\title{
Evaluation of CD40 and CD80 receptors in the colonic mucosal membrane of children with inflammatory bowel disease
}

\author{
Barbara Kamińska', Izabela Roszko-Kirpsza², Piotr Landowski', \\ Agnieszka Szlagatys-Sidorkiewicz', Katarzyna Guzińska-Ustymowicz³, Elżbieta Maciorkowska² \\ ${ }^{1}$ Department of Pediatrics, Pediatric Gastroenterology, Hepatology and Nutrition, Medical University of Gdansk, Poland \\ ${ }^{2}$ Department of Developmental Age Medicine and Paediatric Nursing, Medical University of Bialystok, Poland \\ ${ }^{3}$ Department of Pathomorphology, Medical University of Bialystok, Poland
}

Kaminska B, Roszko-Kirpsza I, Landowski P, Szlagatys-Sidorkiewicz A, Guzinska-Ustymowicz K, Maciorkowska E. Evaluation of CD40 and CD80 receptors in the colonic mucosal membrane of children with inflammatory bowel disease. Ann Agric Environ Med. $2015 ; 22(4)$ : $695-699$. doi: $10.5604 / 12321966.1185778$

\begin{abstract}
Introduction. The most prevalent inflammatory bowel diseases (IBD) include ulcerative colitis (UC) and Crohn's disease (CD). Immune processes play a vital role in the etiopathogenesis of these conditions, involving both cellular and humoral response mechanisms. The aim of this study was to quantify CD40- and CD80-positive cells in the biopsy specimens of large intestinal mucosa from children with IBD.

Materials and method. The study comprised 38 children aged between 3-17 years (mean $11.5 \pm 3.7$ years) - 20 boys (52.6\%) and 18 girls (47.4\%). Eighteen patients were diagnosed with UC on the basis of clinical manifestation, endoscopic and histopathological findings. Mean age of this subgroup was $11.55 \pm 4.07$ years. A group of 10 children (mean age 12.30 \pm 2.83 ) diagnosed with CD was also included. The control group comprised 10 IBD-free children (mean age 10.28 \pm 4.07 years). The surface expressions of CD40 and CD80 were analyzed in large intestine mucosa biopsy specimens, fixed in formaldehyde, embedded in paraffin, and cut with a microtome into $4 \mu \mathrm{m}$ slices.

Results. The number of CD40- and CD80-positive cells in the large intestinal mucosa of children with Crohn's disease and ulcerative colitis was significantly higher than in the controls. The highest number of CD40+ and CD80+ cells was observed in the caecal mucosal membrane of Crohn's disease patients and in the rectal mucosa of individuals with ulcerative colitis. Conclusion. IBD is characterized by elevated, segment-specific, expression of CD40 and CD80.
\end{abstract}

\section{Key words}

inflammatory bowel disease, children, CD40, CD 80 receptors

\section{INTRODUCTION}

Ulcerative colitis (UC) and Crohn's disease (CD) are the most prevalent inflammatory bowel diseases (IBD). Immune processes, involving both cellular and humoral response mechanisms, play a pivotal role in the etiopathogenesis of these conditions $[1,2]$.

An increased incidence of IBD in children has been observed for several decades, along with a decrease in patients' age. It is estimated that as many as $15 \%$ of the patients show initial symptoms of the disease during childhood or even in the neonatal period [3]. the peak incidence, however, is observed around the age of 30 . The incidence in pubertal children amounts to 7 per 100,000 , increasing to 12 per 100,000 in the young adulthood, i.e. between $20-29$ years of age [4].

In children, a greater number of the new cases are diagnosed with CD than UC. The clinical manifestation of UC in children is frequently more severe than in adults, with $70-80 \%$ of paediatric patients suffering from pancolitis as early as at the initial diagnosis. Pancolitis, along with the involvement of the upper alimentary tract, is also observed in paediatric $\mathrm{CD}$ patients $[5,6]$.

Address for correspondence: Barbara Kamińska, Department of Pediatrics, Pediatric Gastroenterology, Hepatology and Nutrition, Medical University of Gdansk, Nowe Ogrody 1-6, 80-803 Gdansk, Poland.

E-mail: bkam@gumed.edu.pl

Received: 21 December 2012; accepted: 30 October 2013
Abnormal response of the immune system to an unidentified antigen is reflected by the imbalance between pro-inflammatory and anti-inflammatory factors in mucosal membrane or in the whole intestinal wall. Molecules involved in the initiation of specific immune response include CD40 and CD80. CD40, a $45-50 \mathrm{kDa}$ molecule, is a component of the CD40/CD40L pathway. It is classified as the type I transmembrane protein, which belongs to the TNF receptor superfamily. CD40 is expressed both on the surface of immune cells, including B lymphocytes, monocytes, macrophages and dendritic cells, and on the surface of non-immune cells: endothelial, epithelial and mesenchymal cells (fibroblasts), and thrombocytes. Binding CD40 to its specific ligand (CD40L) located on T lymphocytes causes the activation of B lymphocytes and macrophages; consequently, initiating pro-inflammatory and pro-coagulatory processes.

CD80 is a membrane receptor activated upon binding of CD28 or CTLA-4, i.e. co-receptors present on the surface of CD4+ and CD8+ lymphocytes. Interaction between CD80 and CD28 is necessary for transducing the signal of detecting the antigen by the antigen presenting cells (APCs); otherwise, T lymphocytes become anergic. For that reason, CD80 is expressed on the surface of many APCs, including macrophages, dendritic cells, Langerhans cells, and B lymphocytes.

Interactions within ligand-receptor pairs, such as CD80CD28 or CD40L-CD40, are responsible for transducing 
the signal between $\mathrm{T}$ and $\mathrm{B}$ lymphocytes, and subsequent initiation of immune response. Presentation of CD40 on the surface of APCs or intestinal endothelial cells is associated with the resultant activation of the main histocompatibility complex (MHC) and co-stimulatory molecules, including CD80 and CD86. The CD40/CD40L-mediated processes are subsequently reflected by the secretion of many cytokines, such as IL-1, IL-6, IL-8, IL-12, and TNF-alpha [7, 8].

The aim of this study was to quantify CD40- and CD80positive cells in the biopsy specimens of large intestine mucosa obtained from children with IBD.

\section{MATERIALS AND METHOD}

The study comprised 38 children aged between $3-17$ years (mean $11.5 \pm 3.7$ years) -20 boys (52.6\%) and 18 girls (47.4\%). Eighteen patients were diagnosed with UC on the basis of clinical manifestation, endoscopic and histopathological findings. Mean age of this subgroup was $11.55 \pm 4.07$ years. A group of 10 children (mean age $12.30 \pm 2.83$ ) with CD was also included. The control group comprised 10 IBD-free children (mean age $10.28 \pm 4.07$ years).

The severity and location of endoscopic changes were verified in all studied children; additionally, the histological examination of the obtained samples was performed and disease activity assessed. In the case of children with UC, the above-mentioned clinical variables were determined on the basis of the Rachmilewitz [9] endoscopic index, Prague classification, Geboes index, and PUCAI scale, respectively $[6,9,10,11,12]$. Endoscopic activity (SES-CD - Simple Endoscopic Score for Crohn's Disease), localization of the lesions (Montreal Scale), and the activity of the disease (PCDAI) were evaluated for patients with CD $[13,14,15]$.

The expressions of CD40 and CD80 on cell surfaces were analyzed in large intestinal mucosal biopsy specimens, fixed in formaldehyde, embedded in paraffin, and cut with a microtome into $4 \mu \mathrm{m}$ slices. The slices were deparaffinized in xylene, and hydrated in alcohol series. The antigens were exposed to the antibodies to CD40 and CD80 in the presence of citrate $(\mathrm{pH}=6.0)$ or TrisEDTA $(\mathrm{pH}=9.0)$ buffers, respectively, in a water bath $\left(20 \mathrm{~min}\right.$ at $\left.97^{\circ} \mathrm{C}\right)$. The activity of endogenous peroxidase was blocked by $10-\mathrm{min}$ incubation with $3 \%$ hydrogen peroxide. Subsequently, the slices were incubated overnight at $4{ }^{\circ} \mathrm{C}$, with mouse antiCD40 monoclonal antibody (1:25 dilution, R\&D Systems), or with rabbit anti-CD80 monoclonal antibody (1:250 dilution, Abcam). The reaction was developed with biotinylated goat anti-rabbit and anti-mouse secondary antibodies conjugated with horseradish peroxidase (LSAB+, DAKO, Poland). Colour reaction for peroxidase was developed with $\mathrm{DAB}$ chromogene (DAKO, Poland). The number of CD40- or CD80-positive cells in large intestinal mucosa was expressed as the mean of the 3 high-power fields of vision $(400 \mathrm{x})$.

Statistical analysis of results was conducted with the Statistica 2007 package. Due to the small size of analyzed groups, the normal distribution of studied variables was verified with the Shapiro-Wilk test. Normally distributed variables in 2 independent groups were compared with the Student's t-test. In the case of not normally distributed variables, the Mann-Whitney U-test was used for the comparisons of 2 independent groups, while more than 2 independent groups were compared with the Kruskal-
Wallis test. The values of dependent, not normally distributed variables were compared with the Wilcoxon test. Mutual relationships between analyzed variables were analyzed with the Spearman's coefficients of rank correlation. The statistical significance was set at $\mathrm{p}<0.05$.

\section{RESULTS}

Immunohistochemical analysis revealed that the intestinal mucosa of patients with UC showed significantly stronger superficial expression of CD40 than in the controls in all 3 analyzed segments: caecum $(\mathrm{p}=0.048)$, sigmoid colon $(\mathrm{p}=0.00009)$, and rectum $(\mathrm{p}=0.001)$. Mean number of CD40+ cells in the caecal mucosa of UC patients ranged between 15-40 per 3 high-power fields (median: 40), compared to 1-40 in the controls (median: 2). The mean number of CD40+ cells in the sigmoid colon mucosa of UC patients ranged between 30-80 (median: 60; Fig. 1), being markedly lower in the controls (10-25 cells, median: 10). Also, the mean number of CD40+ cells in the rectal mucosa of UC children (15-40, median: 30) was higher than in the controls (1-20, median: 10).

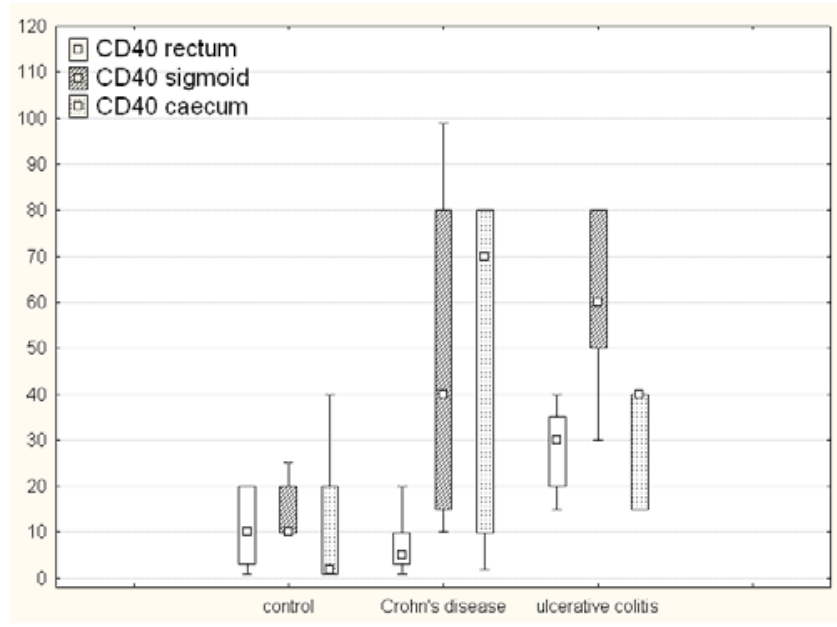

Figure 1. Infiltration of CD40+ cells into the sigmoid mucosa of children with ulcerative colitis $(400 \mathrm{x})$

The immunohistochemically-determined number of CD $40+$ cells in caecal and sigmoid specimens of CD patients was different from the controls ( $p=0.015$ and $p=0.05$, respectively); no significant intergroup differences were observed in the case of rectal specimens $(p=0.48)$. Mean number of CD $40+$ cells in the caecal mucosa of CD patients ranged between $2-80$ per 3 high-power fields (median: 70 ), as compared to $1-40$ cells in the controls (median: 2 ). The number of positive cells in the sigmoid mucosa of CD patients and the controls ranged from 10-99 (median: 40) and 10-25 cells (median: 10), respectively. The mean number of CD40+ cells per 3 high-power fields of rectal specimens ranged between 1-20 in each group (median of 5 and 10 in CD and control children, respectively).

Comparative analysis of the number of CD40+ cells in the large intestinal mucosa of children with $U C$ and CD revealed segment-specific variability. While the patients with UC showed significantly higher proportion of CD40-positive cells in rectal specimens $(\mathrm{p}<0.0001)$, no significant intergroup differences were documented in the case of sigmoid $(\mathrm{p}=0.33)$ and caecal mucosa ( $\mathrm{p}=0.48$; Fig. 2). 


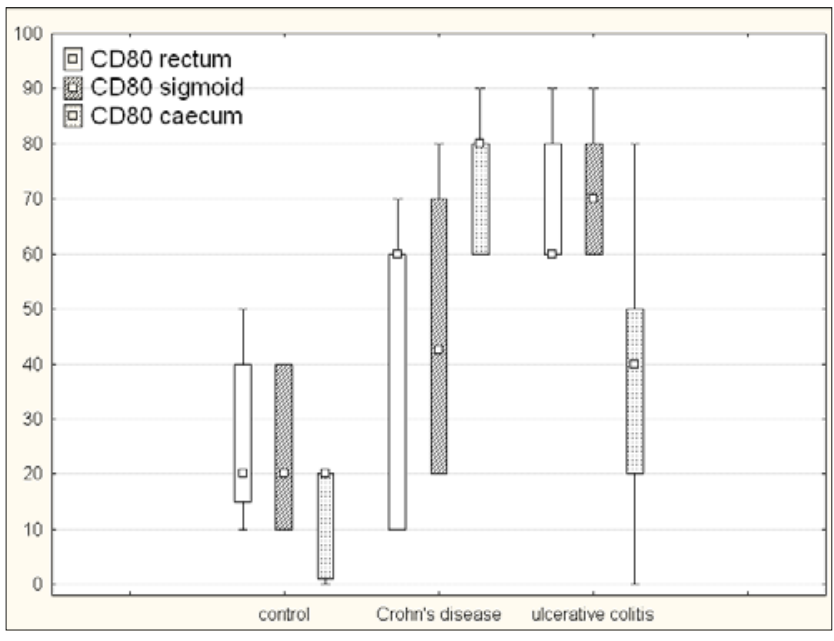

Figure 2. Number of CD40+ cells in the mucosa of large intestine of the studied patients

Compared to the controls, patients with UC were characterized by significantly higher superficial expression of CD80 on caecal $(\mathrm{p}=0.067)$, sigmoid $(\mathrm{p}<0.001)$, and rectal epithelial cells $(\mathrm{p}<0.009)$. The number of CD80-positive cells in the caecal mucous membrane of UC patients ranged between $0-80$ per 3 high-power fields (median: 40), as compared to $0-20$ cells (median: 20 ) in the controls. The number of CD80+ cells in the sigmoid colon of UC patients ranged between 10-90 (median: 70); markedly lower values were found in the controls (10-40 cells, median: 20$)$. The number of CD80+ cells in the rectal mucosa of children with UC ranged between 15-90 (median: 60), and was significantly higher than in the controls (10-50 cells, median: 20). Compared to the controls, children with CD were characterized by a significantly higher number of CD80+ cells in the caecal mucosa $(\mathrm{p}<0.0004)$; however, these 2 groups did not differ significantly in terms of the number of positive cells in sigmoid and rectal specimens $(p=0.07$ and $p=0.37$, respectively). The mean number of $\mathrm{CD} 80+$ cells in the caecal mucosa of CD patients ranged between 60-90 per 3 highpower fields (median: 70; Fig. 3), while the corresponding

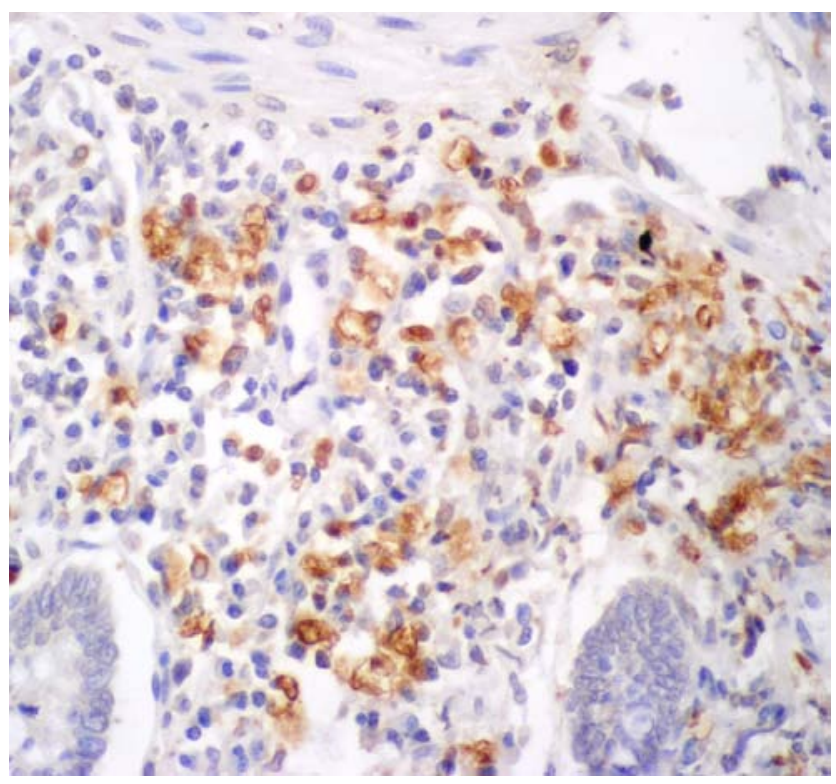

Figure 3. Infiltration of $C D 40+$ in sigmoid mucosa in children with ulcerative colitis

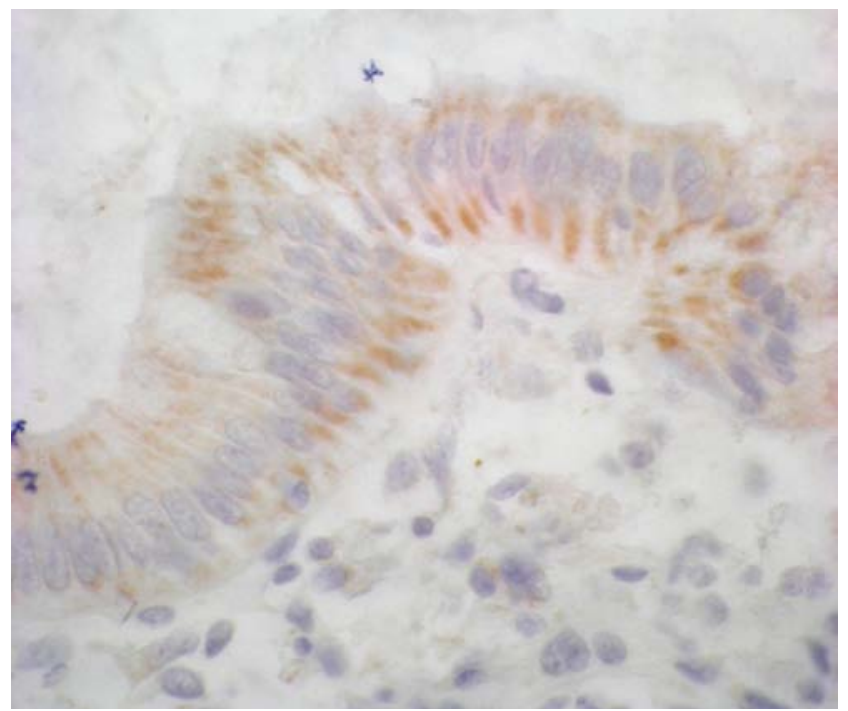

Figure 4. Infiltration of CD80+ cells in cecal mucosa in children with Crohn Disease

values in the controls ranged between $0-20$ (median: 20). The number of CD $80+$ cells in the sigmoid mucous membrane ranged between $20-80$ (median: 42.5 ) in CD patients, and from 10-40 (median: 20) in the controls, while the numbers of CD80-positive cells in rectal specimens of these 2 groups were equal to 10-70 (median: 60) and 10-50 (median: 20), respectively.

Comparative analysis of the number of CD80+ cells in the large intestinal mucosa of UC and CD patients revealed that the latter group was characterized by a significantly higher count of positive cells in caecal specimens $(p<0.002)$. In contrast, these 2 groups did not differ significantly with regards to the number of CD80+ cells in sigmoid $(\mathrm{p}=0.1)$ and rectal specimens $(\mathrm{p}=0.06$; Fig. 4$)$.

\section{DISCUSSION}

IBD represents a heterogeneous group of chronic inflammatory bowel diseases with unknown, most probably multifactorial, pathogenesis. This likely involves genetic predispositions, exposure to environmental factors, bacteria and cellular allergens, and deregulation of the intestinal immune system. The impaired immune response to gastrointestinal allergens, such as microorganisms or dietary components, is postulated to lead to the abnormal inflammatory response and the development of non-specific inflammation of the intestine $[1,16]$.

During IBD, the immune balance of colonic mucosa is disturbed and the focal signs of inefficient immune response may be observed. Altered adhesion to endothelium of microvessels is reflected by the extravasation of $\mathrm{T}$ and $\mathrm{B}$ cells, leading to the concentration of a high number of plasmatic cells, as well as macrophages and neutrophils, in the mucosal membrane [17].

Mediators released by the infiltrating mononuclear cells cause injury to mucosal membrane of individuals with $\mathrm{UC}$, or to the deeper layers of the intestinal wall of CD patients. In the course of IBD, the signs of enhanced cytokine synthesis by immunomodulatory cells are observed within the mucosal membrane. Macrophages correspond to $15 \%$ of mononuclear cells present on the lamina propria of non- 
inflamed mucosa. In contrast, an increased number of monocytes and macrophages can be found in the inflamed mucosa as a result of migration into or through the mucosal lamina propria [14].

Studies conducted by Danese et al. [18], Battaglia et al. [19], Polese et al. [20], and Vogel et al. [21], using immunohistochemical methods to analyze the distribution of CD40 within the normal mucosa of the large intestine, revealed the expression of this molecule only in solitary mononuclear cells of the mucosal lamina propria, as well as in the endothelial cells of submucosal microcirculation. In contrast, the expression of CD40 was not detected in the cells of mucosal muscle layer, and in the mesenchymal cells of submucosal membrane.

Also in the presented study, the number of CD40-positive cells in the control specimens was low (median number: 2 in the cecum, and 10 for both the sigmoid colon and rectum).

Many authors have noted the marked increase in the number of CD40-positive cells in the inflamed ileal or colonic mucosa of IBD patients $[19,22]$. Using immunohistochemical techniques, the CD40-positive cells were identified as B cells, as well as endothelial cells, macrophages, and fibroblasts. Battaglia et al. [19], Polese et al. [20], and Vogel et al. [21] confirmed immunohistochemically that the actively inflamed intestinal wall of UC or CD patients contains mononuclear, endothelial, and mesenchymal cells showing strong expression of CD40, scattered both in the mucosal and submucosal membrane $[19,20,21]$. In the study by Polese et al. [20], the number of CD40-positive cells in the colonic mucosa of UC patients was markedly elevated and correlated with disease activity determined on the basis of clinical, endoscopic, and histological criteria. There were statistically significant differences between UC and IBD patients $(\mathrm{p}<0.005)$, even when these 2 groups were compared during the period of remission $(\mathrm{p}<0.05)$. In contrast, the number of CD40-positive cells in CD and UC cells was similar. Mean percentage of CD40+ cells per one field of vision of the mucous membrane of the large intestine was $21 \pm 11 \%$ in UC, $24 \pm 9 \%$ in CD, and $7 \pm 7 \%$ in IBD. Moreover, it was revealed that during severe inflammation, the expression of CD40 on the cells of intestinal mucosa was significantly stronger, compared to moderately severe inflammation [23].

In the current study, the number of CD40-positive cells both in UC and CD patients was higher than in the controls. In the case of UC patients, a significantly higher number of positive cells was documented in all 3 analyzed segments of the large intestine; whereas, significant differences were observed in CD patients in caecal and sigmoid specimens. The comparative analysis of the number of CD40-positive cells on the surface of large intestinal mucosa in both groups of patients revealed a higher number of these cells in UC patients, but only in the case of rectal specimens. In the study by Sawada-Hase et al.[24], the location of CD40positive cells in the intestinal mucosa of CD patients was analyzed using double-labeled immunohistochemistry. In the case of inflamed large intestinal mucosa, the expression of CD40 was revealed on a large number of mononuclear cells infiltrating the mucosal lamina propria. Additionally, these cells showed the superficial expression of CD68 (a marker of mature tissue macrophages); in contrast, there was no expression of CD40 and CD20 (an identification marker of $B$ lymphocytes). Therefore, the CD40-positive cells present in the large intestinal mucosa of $\mathrm{CD}$ patients were identified as tissue macrophages. Similarly to previous studies, the number of CD40+ cells in normal mucosa was very low [24].

In both groups of patients participating in the presented study, the CD40-positive cells were also located in the lamina propria of large intestinal mucosa, as well as on the surface of epithelial cells of glandular tubules. Intestinal epithelial cells constitute the key link between the environment and the host. They are involved in the regulation of inflammatory response of intestinal mucosal membrane to antigens. Moreover, they function as APCs for various subgroups of $\mathrm{T}$ cells, and play a role in innate immune response, secreting a panel of cytokines and chemokines. The involvement of intestinal epithelium was shown by Borcherding et al. [25], who revealed the presence of CD40 on the surface of intestinal epithelial cells in the mucosal specimens obtained from CD and UC patients. The intraepithelial expression of CD40 was documented in the majority of patients with caecal and colonic inflammation associated with CD or active UC. The presence of CD40-positive cells in the epithelium of inflamed mucosa was observed both in the crypts and villi of the caecum, as well as in the colonic crypts, and on the surface of colonic epithelium. The expression of CD40 was polarized at the subcellular level, being limited to the basolateral membrane of epithelial cells. Both in CD and UC patients, the inflammatory infiltrate of CD40-positive cells was found to be significantly enhanced solely in the mucosal areas involved in the inflammatory process. In contrast, no intestinal epithelial expression of CD40 was documented in ileal and colonic biopsy specimens obtained from patients in remission. Similarly, the expression of CD40 was nearly absent on the intestinal epithelial cells of the controls. The intestinal epithelial expression of CD40 was observed in all patients with ileitis (100\%) and in the majority of patients with the inflammation of large intestine $(85 \%)$, who were in the active phase of CD; in contrast, the expression was lower in the relevant sites of patients in remission (20\% of CD40-positive ileal cells, and 5\% of colonic cells) [26]. Similar findings were documented in UC patients $(92.5 \%$ of the positive cells in the active phase and $3.70 \%$ in remission). These results confirmed the epithelial expression and modulation of CD40 in mucosal membrane affected with IBD, as well as the pro-inflammatory activity of intestinal epithelial cells [27]. Battaglia et al. [19] observed that CD40-positive cells are absent in the biopsy specimens of microscopically-normal mucosa obtained from the sites distant from those involved in the inflammatory process [19].

Mucosal biopsy specimens obtained from the large intestine of children with IBD showed a significantly higher density of CD40+ cells than the control specimens. Furthermore, the density of CD40-positive cells in the colonic biopsies of IBD children without the histological signs of inflammation was higher, although not markedly, than in the control non-IBD group. In contrast, the count of CD40+ in the colon of CD children was markedly elevated, compared to the controls. Moreover, the CD children showed a tendency for a higher density of CD40+ cells in the colon, compared to UC patients (mean rank 10.7 vs. 8.7) [28].

Maerten et al. [29] and Rugtveit et al. [30] revealed that the expression of CD80 in normal ileal and large intestinal mucosa is low. In contrast, the percentage of CD80+ cells is significantly increased in the inflamed mucosa. Most immunohistochemically-identified cells with CD80 expression were located in the mucosal lamina propria 
and in the submucosal membrane. Macrophages located in the subepithelial region represented the highest fraction of CD80+ cells. The cells showing the expression of CD80 were observed on the surface of macrophages, both present within the lymphatic aggregates and scattered in the mucosal lamina propria of the large intestine of IBD patients (or the ileum of individuals with $\mathrm{CD}$ ).

In the presented study, the number of CD80-positive cells in the caecum, sigmoid colon, and rectum of UC patients was significantly higher than in the controls. Moreover, the number of CD80+ cells in the mucosa of various large intestinal segments of CD children were analysed. Compared to the controls, this group was characterized by a significantly higher count of positive cells in the caecum, while no significant differences were documented in the case of sigmoid and rectal specimens. Comparative analysis of the mucosal expression of CD80 in UC and CD patients revealed that the latter group had a significantly higher number of positive cells in the caecum; in contrast, no significant intergroup differences were documented in the case of the sigmoid colon and the rectum.

\section{CONCLUSIONS}

1. The number of CD40- and CD80-positive cells in the mucous membrane of the large intestine of children with Crohn's disease and ulcerative colitis is significantly higher than in the controls.

2. The highest number of CD40+ and CD80+ cells is observed in the caecal mucosal membrane of Crohn's disease patients and in the rectum of individuals with ulcerative colitis.

\section{REFERENCES}

1. Baumgart DC, Carding SR. Inflammatory bowel disease: cause and immunobiology. Lancet. 2007; 369: 1627-1640.

2. Polese L, Angriman I, Scarpa M, Norberto L, Sturniolo GC, Cecchetto A, et al. Role of CD40 and B7 costimulators in inflammatory bowel diseases. Acta Biomed. 2003; 74 Suppl 2: 65-70.

3. IBD Working Group of the European Society for Paediatric Gastroenterology, Hepatology and Nutrition. Inflammatory bowel disease in children and adolescents: recommendations for diagnosis - the Porto criteria. J Pediatr Gastroenterol Nutr. 2005; 41(1): 1-7.

4. Nieuwenhuis EE, Escher JC. Early onset IBD: what's the difference? Dig Liver Dis. 2008; 40(1): 12-15.

5. Bousvaros A, Antonioli DA, Colletti RB, Dubinsky MC, Glickman JN, Gold BD, et al. Differentiating ulcerative colitis from Crohn disease in children and young adults: report of a working group of the North American Society for Pediatric Gastroenterology, Hepatology, and Nutrition and the Crohn's and Colitis Foundation of America. J Pediatr Gastroenterol Nutr. 2007; 44(5): 653-674.

6. Freeman HJ. Comparison of longstanding pediatric-onset and adultonset Crohn's disease. J Pediatr Gastroenterol Nutr. 2004; 39(2): $183-186$.

7. Grzybowska-Chlebowczyk U, Pająk J, Woś H, Gabryel B, Więcek SMK. Ocena stężenia TNF- $\alpha$ w surowicy dzieci z nieswoistymi zapaleniami jelit. Przegl Gastroenterol. 2008; 3: 149-153 (in Polish).

8. Gruchlik A, Chodurek EZD. Rola miofibroblastów w chorobach zapalnych jelit i procesach nowotworzenia. Przegl Gastroenterol. 2011; 6: 353-358 (in Polish).

9. Rachmilewitz D. Coated mesalazine (5-aminosalicylic acid) versus sulphasalazine in the treatment of active ulcerative colitis: a randomised trial. BMJ. 1989; 298(6666): 82-86.

10. Turner D, Otley AR, Mack D, Hyams J, de Bruijne J, Uusoue K, et al. Development, validation, and evaluation of a pediatric ulcerative colitis activity index: a prospective multicenter study. Gastroenterology. 2007; 133(2): 423-432.

11. Satsangi J, Silverberg MS, Vermeire S, Colombel JF. The Montreal classification of inflammatory bowel disease: controversies, consensus, and implications. Gut. 2006; 55(6): 749-753.

12. Bernstein CN, Fried M, Krabshuis JH, Cohen H, Eliakim R, Fedail S, et al. World Gastroenterology Organization Practice Guidelines for the diagnosis and management of IBD in 2010. Inflamm Bowel Dis. 2010; 16(1): 112-124.

13. Daperno M, D’Haens G, Van Assche G, Baert F, Bulois P, Maunoury $\mathrm{V}$, et al. Development and validation of a new, simplified endoscopic activity score for Crohn's disease: the SES-CD. Gastrointest Endosc. 2004; 60(4): 505-512.

14. Levine A, Griffiths A, Markowitz J, Wilson DC, Turner D, Russell RK, et al. Pediatric modification of the Montreal classification for inflammatory bowel disease: the Paris classification. Inflamm Bowel Dis. $2011 ; 17(6)$ : 1314-1321.

15. Turner D, Griffiths AM, Walters TD, Seah T, Markowitz J, Pfefferkorn $\mathrm{M}$, et al. Appraisal of the pediatric Crohn's disease activity index on four prospectively collected datasets: recommended cutoff values and clinimetric properties. Am J Gastroenterol. 2010; 105(9): 2085-2092.

16. Grzybowska K. Udział układu immunologicznego w patogenezie nieswoistych zapaleń jelit u dzieci. Nowa Pediatria. 2003; 1: 81-84 (in Polish).

17. Brown SJ, Mayer L. The immune response in inflammatory bowel disease. Am J Gastroenterol. 2007; 102(9): 2058-2069.

18. Danese S, Sans M, Fiocchi C. The CD40/CD40L costimulatory pathway in inflammatory bowel disease. Gut. 2004; 53(7): 1035-1043.

19. Battaglia E, Biancone L, Resegotti A, Emanuelli G, Fronda GR, Camussi G. Expression of CD40 and its ligand, CD40L, in intestinal lesions of Crohn's disease. Am J Gastroenterol. 1999; 94(11): 3279-3284.

20. Polese L, Angriman I, Cecchetto A, Norberto L, Scarpa M, Ruffolo C, et al. The role of CD40 in ulcerative colitis: histochemical analysis and clinical correlation. Eur J Gastroenterol Hepatol. 2002; 14(3): 237-241.

21. Vogel JD, West GA, Danese S, De La Motte C, Phillips MH, Strong SA, et al. CD40-mediated immune-nonimmune cell interactions induce mucosal fibroblast chemokines leading to T-cell transmigration. Gastroenterology. 2004; 126(1): 63-80.

22. Liu Z, Colpaert S, D'Haens GR, Kasran A, de Boer M, Rutgeerts P, et al. Hyperexpression of CD40 ligand (CD154) in inflammatory bowel disease and its contribution to pathogenic cytokine production. J Immunol. 1999; 163(7): 4049-4057.

23. Carlsen HS, Yamanaka T, Scott H, Rugtveit J, Brandtzaeg P. The proportion of CD40+ mucosal macrophages is increased in inflammatory bowel disease whereas CD40 ligand (CD154)+ T cells are relatively decreased, suggesting differential modulation of these costimulatory molecules in human gut lamina propria. Inflamm Bowel Dis. 2006; 12(11): 1013-1024.

24. Sawada-Hase N, Kiyohara T, Miyagawa J, Ueyama H, Nishibayashi H, Murayama Y, et al. An increased number of CD40-high monocytes in patients with Crohn's disease. Am J Gastroenterol. 2000; 95(6): 1516-1523.

25. Borcherding F, Nitschke M, Hundorfean G, Rupp J, von Smolinski $\mathrm{D}$, Bieber K, et al. The CD40-CD40L pathway contributes to the proinflammatory function of intestinal epithelial cells in inflammatory bowel disease. Am J Pathol. 2010; 176(4): 1816-1827.

26. Danese S, Sans M, Scaldaferri F, Sgambato A, Rutella S, Cittadini A, et al. TNF-alpha blockade down-regulates the CD40/CD40L pathway in the mucosal microcirculation: a novel anti-inflammatory mechanism of infliximab in Crohn's disease. J Immunol. 2006; 176(4): 2617-2624.

27. Hart AL, Al-Hassi HO, Rigby RJ, Bell SJ, Emmanuel AV, Knight SC, et al. Characteristics of intestinal dendritic cells in inflammatory bowel diseases. Gastroenterology. 2005; 129(1): 50-65.

28. Perminow G, Reikvam DH, Lyckander LG, Brandtzaeg P, Vatn MH, Carlsen HS. Increased number and activation of colonic macrophages in pediatric patients with untreated Crohn's disease. Inflamm Bowel Dis. 2009; 15(9): 1368-1378.

29. Maerten P, Liu Z, Ceuppens JL. Targeting of costimulatory molecules as a therapeutic approach in inflammatory bowel disease. BioDrugs. 2003; 17(6): 395-411.

30. Rugtveit J, Bakka A, Brandtzaeg P. Differential distribution of B7.1 (CD80) and B7.2 (CD86) costimulatory molecules on mucosal macrophage subsets in human inflammatory bowel disease (IBD). Clin Exp Immunol. 1997; 110(1): 104-113. 